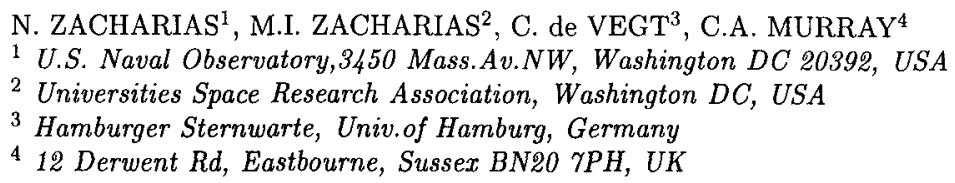

The Second Cape Photographic Catalog (CPC2) contains 276,131 stars covering the entire Southern Hemisphere in a 4-fold overlap pattern. Its mean epoch is 1968 , which makes it a key catalog for proper motions. A new reduction of the 5687 plates using on average 40 Hipparcos stars per plate has resulted in a vastly improved catalog with a positional accuracy of about 40 mas (median value) per coordinate, which comes very close to the measuring precision. In particular, for the first time systematic errors depending on magnitude and color can be solved unambiguously and have been removed from the catalog. In combination with the Tycho Catalogue (mean epoch 1991.25) and the upcoming U.S. Naval Observatory CCD Astrograph Catalog (UCAC) project proper motions better than $2 \mathrm{mas} / \mathrm{yr}$ can be obtained. This will lead to a vastly improved reference star catalog in the Southern Hemisphere for the final Astrographic Catalogue (AC) reductions, which will then provide proper motions for millions of stars when combined with new epoch data. These data then will allow an uncompromised reduction of the southern Schmidt surveys on the International Celestial Reference System (ICRS).

\title{
ASTROMETRY OF POSS-II PLATES USING TYCHO
}

\author{
B. BUCCIARELLI ${ }^{1}$, J.E. MORRISON ${ }^{2}$, B.J. MCLEAN ${ }^{2}$, C.R. STURCH ${ }^{2,3}$ \\ 1 Astronomical Observatory of Torino, Italy \\ 2 Space Telescope Science Institute, USA \\ 3 Computer Science Corporation at STScI
}

We present a first attempt to compute an astrometric solution of POSS-II plates using TYCHO stars as the reference catalogue. Also, we have made use of a special catalogue of stars observed with the Carlsberg Meridian Circle in La Palma between 1994 and 1995, brought into the HIPPARCOS reference system. Such a catalogue consists of a double strip along the equator, fairly dense (approximately 10 stars per square degree), faint (average magnitude 12.5), with an average error at epoch of $\sim 150$ mas per coordinate. Since the limiting magnitude of TYCHO stars is about 11.5, almost exactly one magnitude less than the CAMC special catalogues' average, the latter has served as a very useful external comparison, although a more interesting magnitude range would have been 14-15. In this respect, we note that the Schmidt plates under study cover a large area $(6.4 \times 6.4$ degree) and have fairly long exposures $(\sim 60 \mathrm{~min})$; therefore, a possible magnitude dependence of the model needs to be carefully investigated. The results shown in the table above, which were obtained using a 2nd order polynomial in conjunction with a mask method using 5 plates only are very promising. The average external error over the entire plate area is about 0.2 arsec per coordinate, after the CAMC error have been subtracted.

\begin{tabular}{|c|c|cc|c|cc|}
\hline & \multicolumn{3}{|c|}{ TYCHO rms (arsec) } & \multicolumn{2}{c|}{ CAMC rms (arsec) } \\
\hline Plate ID & TYCHO stars & Ra & Dec & CAMC stars & Ra & Dec \\
\hline A094 & 1019 & 0.21 & 0.20 & 358 & 0.30 & 0.30 \\
A096 & 1057 & 0.22 & 0.20 & 346 & 0.29 & 0.32 \\
A0OZ & 586 & 0.18 & 0.16 & 358 & 0.24 & 0.26 \\
A0NR & 509 & 0.14 & 0.15 & 398 & 0.26 & 0.25 \\
A15M & 379 & 0.16 & 0.16 & 346 & 0.25 & 0.27 \\
\hline
\end{tabular}

